\title{
Properties of the Quasi-Conformal Curvature Tensor of Kähler-Norden Manifolds
}

\author{
Uday Chand De and Pradip Majhi
}

\begin{abstract}
The object of the present paper is to study quasi-conformally flat and parallel quasi-conformal curvature tensor of a Kähler-Norden manifold. Besides this we also study quasi-conformally semisymetric Kähler-Norden manifolds. Finally, we mention an example to verify a Theorem of our paper.
\end{abstract}

\section{INTRODUCTION}

An anti-Kähler or Kähler-Norden manifold means a triple $\left(M^{n}, J, g\right)$ which consists of a smooth manifold $M^{n}$ of dimension $n=2 m$, an almost complex structure $J$ and an anti-Hermitian metric $g$ such that $\nabla J=0$ where $\nabla$ is the Levi-Civita connection of $g$. The metric $g$ is called anti-Hermitian if it satisfies $g(J X, J Y)=-g(X, Y)$ for all vector fields $X$ and $Y$ on $M^{2 m}$. Then the metric $g$ has necessarily a neutral signature $(m, m)$ and $M^{2 m}$ is a complex manifold and there exists a holomorphic metric on $M^{2 m}$ [1]. This fact gives us some topological obstructions to an anti-Kähler manifold, for instance, all its odd Chern numbers vanish because its holomorphic metric gives us a complex isomorphism between the complex tangent bundle and its dual and a compact simply connected Kähler manifold cannot be antiKähler because it does not admit a holomorphic metric.

The conditions of the semisymmetry and pseudosymmetry type for the Riemann, Ricci and Weyl curvature tensors of Kählerian and para-Kählerian manifolds were studied in the papers $[9,10,11,12]$ and many others. In the present paper we extend the result of Sluka [5] in a Kähler-Norden manifold. In [4] Sluka constructed some examples of holomorphically projectively flat as well as semisymmetric and locally symmetric Kähler-Norden manifolds. The present paper is organized as follows:

After preliminaries in section 3, we study quasi-conformally flat KählerNorden manifolds. In section 4, we consider parallel quasi-conformal KählerNorden manifolds. In section 5, we study quasi-conformally semisymmetric

2010 Mathematics Subject Classification. Primary: 53C15; Secondary: 53C56, 53C50.

Key words and phrases. Kähler-Norden manifold, quasi-conformally flat, parallel quasiconformal curvature tensor and quasi-conformally semisymmetric. 
Kähler-Norden manifolds. Finally, we mention an example to verify the Theorem 4.1.

\section{Preliminaries}

By a Kählerian manifold with Norden metric (Kähler-Norden in short) [2] we mean a triple $(M, J, g)$, where $M$ is a connected differentiable manifold of dimension $n=2 m, J$ is a $(1,1)$-tensor field and $g$ is a pseudo-Riemannian metric on $M$ satisfying the conditions

$$
J^{2}=-I, \quad g(J X, J Y)=-g(X, Y), \quad \nabla J=0
$$

for every $X, Y \in \chi(M)$ is the Lie algebra of vector fields on $M$ and $\nabla$ is the Levi-Civita connection of $g$.

Let $(M, J, g)$ be a Kähler-Norden manifold. Since in dimension two such a manifold is flat, we assume in the sequel that $\operatorname{dim} M \geq 4$. Let $\mathcal{R}(X, Y)$ be the curvature operator $\left[\nabla_{X}, \nabla_{Y}\right]-\nabla_{[X, Y]}$ and let $\mathcal{R}$ be the RiemannChristoffel curvature tensor, $R(X, Y, Z, W)=g(\mathcal{R}(X, Y) Z, W)$. The Ricci tensor $S$ is defined as $S(X, Y)=\operatorname{trace}\{Z \longrightarrow \mathcal{R}(Z, X) Y\}$. These tensors have the following properties [1]

$$
\begin{aligned}
\mathcal{R}(J X, J Y) & =-\mathcal{R}(X, Y), \quad \mathcal{R}(J X, Y)=\mathcal{R}(X, J Y), \\
S(J Y, Z) & =\operatorname{trace}\{X \longrightarrow \mathcal{R}(J X, Y) Z\}, \quad S(J X, Y)=S(J Y, X), \\
S(J X, J Y) & =-S(X, Y) .
\end{aligned}
$$

Let $Q$ be the Ricci operator. Then we have $S(X, Y)=g(Q X, Y)$ and

$$
Q Y=-\sum_{i} \epsilon_{i} \mathcal{R}\left(e_{i}, Y\right) e_{i}
$$

where $\left\{e_{1}, e_{2}, \ldots, e_{n}\right\}$ is an orthonormal basis and $\epsilon_{i}$ are the indicators of $e_{i}$, $\epsilon_{i}=g\left(e_{i}, e_{i}\right)= \pm 1$. The notion of a quasi-conformal curvature tensor was given by Yano and Sawaki [6]. The quasi-conformal curvature tensor $\tilde{C}$ is defined by

$$
\begin{aligned}
\tilde{C}(X, Y) Z= & a \mathcal{R}(X, Y) Z+b[S(Y, Z) X-S(X, Z) Y+g(Y, Z) Q X \\
& -g(X, Z) Q Y]-\frac{r}{n}\left[\frac{a}{n-1}+2 b\right][g(Y, Z) X-g(X, Z) Y],
\end{aligned}
$$

where $a$ and $b$ are constants and $\mathcal{R}, Q$ and $r$ are Riemannain curvature tensor of type $(1,3)$, the Ricci operator defined by $g(Q X, Y)=S(X, Y)$ and the scalar curvature, respectively. If $a=1$ and $b=-\frac{1}{n-2}$, then (2) takes 
the form

$$
\begin{aligned}
\tilde{C}(X, Y) Z= & \mathcal{R}(X, Y) Z-\frac{1}{n-2}[S(Y, Z) X-S(X, Z) Y+g(Y, Z) Q X \\
& -g(X, Z) Q Y]+\frac{r}{(n-1)(n-2)}[g(Y, Z) X-g(X, Z) Y] \\
= & C(X, Y) Z,
\end{aligned}
$$

where $C$ is the conformal curvature tensor [8]. Thus the conformal curvature tensor $C$ is the particular case of the tensor $\tilde{C}$. For this reason $\tilde{C}$ is called quasi-conformal curvature tensor. A manifold $\left(M^{n}, g\right)(n>3)$ shall be called quasi-conformally flat if $\tilde{C}=0$. It is known [3] that a quasi conformally flat manifold is either conformally flat if $a \neq 0$ or Einstein if $a=0$ and $b \neq 0$. Since they give no restrictions for manifolds if $a=0$ and $b=0$, it is essential for us to consider the case of $a \neq 0$ or $b \neq 0$.

Using (1) and (2) we have

$$
\begin{aligned}
\sum_{i} \epsilon_{i} g\left(\tilde{C}\left(J e_{i}, J Y\right) e_{i}, W\right)= & b\left[2 S(J Y, J W)-r^{*} g(J Y, W)\right] \\
& -\frac{r}{n}\left[\frac{a}{n-1}+2 b\right] g(J Y, J W) .
\end{aligned}
$$

This implies that

$$
\begin{aligned}
\sum_{i} \epsilon_{i} \tilde{C}\left(J e_{i}, J Y\right) e_{i}= & b\left[-2 Q Y-r^{*} J Y\right] \\
& +\frac{r}{n}\left[\frac{a}{n-1}+2 b\right] Y,
\end{aligned}
$$

where $r^{*}$ is the *-scalar curvature, which is defined as the trace of $J Q$. In the above we have applied the identity $\sum_{i} \epsilon_{i} g\left(J e_{i}, e_{i}\right)=0$, which is a consequence of the traceless of $J$.

The holomorphocally projective curvature tensor is defined in the following way $[4,7]$

$$
P(X, Y)=\mathcal{R}(X, Y)-\frac{1}{n-2}\left(X \wedge_{S} Y-J X \wedge_{S} J Y\right),
$$

where the operator $X \wedge_{S} Y$ is defined by

$$
\left(X \wedge_{S} Y\right) Z=S(Y, Z) X-S(X, Z) Y, \quad Z \in \chi(M) .
$$

We notice, for later use, that this tensor has the following properties $P(X, Y, Z, W)=-P(Y, X, Z, W), \quad P(J X, J Y, Z, W)=-P(X, Y, Z, W)$,

$$
\sum_{i} \epsilon_{i} P\left(e_{i}, Y, Z, J e_{i}\right)=0, \quad \sum_{i} \epsilon_{i} P\left(X, Y, e_{i}, e_{i}\right)=0,
$$

A Kähler-Norden manifold $(M, J, g)$ is holomorphically projectively flat if and only if its holomorphically projective curvature tensor $P$ vanishes identically. 
A Riemannian manifold is said to be quasi-conformally semisymmetric if $\mathcal{R}(X, Y) \cdot \tilde{C}=0$, where $\mathcal{R}(X, Y)$ denotes the derivation of the tensor algebra at each point of the manifold for tangent vector fields $X, Y$.

\section{Qusi-CONFORMALLY FLAT KÄHLER-NORDEN MANIFOLDS}

In this section we study qusi-conformally flat Kähler-Norden manifolds, that is, $\tilde{C}(X, Y) Z=0$. Therefore from (3) we obtain

$$
b\left[2 S(J Y, J W)-r^{*} g(J Y, W)\right]=\frac{r}{n}\left[\frac{a}{n-1}+2 b\right] g(J Y, J W),
$$

Using (1) in (8) yields

$$
b\left[-2 S(Y, W)-r^{*} g(J Y, W)\right]=-\frac{r}{n}\left[\frac{a}{n-1}+2 b\right] g(Y, W),
$$

Contracting (9) with respect to the pair of arguments $Y, W$ (that is, taking $Y=W=e_{i}$ into (9), multiplying by $\epsilon_{i}$ and summing up over $i \in\{1, \ldots, n\}$ ), we have

$$
-2 b r=-\frac{r}{n}\left[\frac{a}{n-1}+2 b\right] n
$$

This implies

$$
-\frac{a}{n-1} r=0 .
$$

Since $a \neq 0$, then from (11) we obtain

$$
r=0 .
$$

Again using (12) in (9) we obtain

$$
S(Y, W)=-\frac{r^{*}}{2 b} g(J Y, W) .
$$

Using (12), (13) in (2) we have

$$
\begin{aligned}
\mathcal{R}(X, Y) Z= & -\frac{r^{*}}{2 a}[-g(J Y, Z) X+g(J X, Z) Y \\
& -g(Y, Z) J X+g(X, Z) J Y] .
\end{aligned}
$$

Also holomorphically projectively flatness implies from (5)

$$
\begin{aligned}
\mathcal{R}(X, Y) Z= & \frac{1}{n-2}[S(Y, Z) X-S(X, Z) Y \\
& -S(J Y, Z) J X+S(J X, Z) J Y] .
\end{aligned}
$$

Therefore from (13) and (15) it follows that

$$
\begin{aligned}
\mathcal{R}(X, Y) Z=\frac{r^{*}}{2 b(n-2)}[-g(J Y, Z) X & +g(J X, Z) Y-g(Y, Z) J X \\
& +g(X, Z) J Y] .
\end{aligned}
$$


From equations (14) and (16) we obtain $r^{*}[a+(n-2) b]=0$. Now, $r^{*}[a+$ $(n-2) b]=0$ implies either $r^{*}=0$ or, $a+(n-2) b=0$. If $a+(n-2) b=0$, then putting this into (2), we get $\tilde{C}(X, Y) Z=a C(X, Y) Z$. So the quasiconformally flatness and conformally flatness are equivalent in this case. Thus in view of the above result we can state the following:

Theorem 3.1. If a quasi-conformally flat Kähler-Norden manifold is holomorphically projectively flat, then quasi-conformally flatness and conformally flatness are equivalent provided $r^{*} \neq 0$.

Corollary 3.1. The Ricci tensor and curvature tensor of a quasi-conformally flat Kähler-Norden manifold $(M, J, g)$ have the shapes (13) and (14), respectively.

\section{Kähler-Norden manifolds $(M, J, g)$ With PARAllel QUASI-CONFORMAL CURVATURE TENSOR}

Assume that the quasi-conformal curvature tensor of a Kähler-Norden manifold is parallel, that is, $\nabla \tilde{C}=0$. From (3) we have

$$
\begin{aligned}
\sum_{i} \epsilon_{i} g\left(\tilde{C}\left(J e_{i}, J Y\right) e_{i}, W\right)= & b\left[2 S(J Y, J W)-r^{*} g(J Y, W)\right] \\
- & \frac{r}{n}\left[\frac{a}{n-1}+2 b\right] g(J Y, J W),
\end{aligned}
$$

where $r^{*}$ is the $*$-scalar curvature, which is defined as the trace of $J Q$. Taking covariant differentiation of (17) and our assumption yields

$$
\begin{aligned}
0=b\left[-2\left(\nabla_{Z} S\right)(Y, W)\right. & \left.-d r^{*}(Z) g(J Y, W)\right] \\
& +\frac{d r(Z)}{n}\left[\frac{a}{n-1}+2 b\right] g(Y, W),
\end{aligned}
$$

since $S(J Y, J W)=-S(Y, W)$ and $g(J Y, J W)=-g(Y, W)$.

Contracting (18) with respect to the pair of arguments $Y, W$ (that is, taking $Y=W=e_{i}$ into (18), multiplying by $\epsilon_{i}$ and summing up over $i \in\{1, \ldots, n\})$, we have

$$
-2 b d r(Z)+\frac{d r(Z)}{n}\left[\frac{a}{n-1}+2 b\right] n=0 .
$$

Since $a \neq 0$, then (19) implies

$$
d r(Z)=0 .
$$

Using (20) in (18) we have

$$
\left(\nabla_{Z} S\right)(Y, W)=-\frac{1}{2} d r^{*}(Z) g(J Y, W) .
$$

Putting $Y=J Y$ in (21) we obtain

$$
\left(\nabla_{Z} S\right)(J Y, W)=\frac{1}{2} d r^{*}(Z) g(Y, W) .
$$


Contracting (22) with respect to the pair of arguments $Y, W$ (that is, taking $Y=W=e_{i}$ into (22), multiplying by $\epsilon_{i}$ and summing up over $i \in\{1, \ldots, n\})$, we have

$$
d r^{*}(Z)=0 \text {. }
$$

Again using (20) and (23) in (18) yields

$$
\left(\nabla_{Z} S\right)(Y, W)=0 .
$$

In view of (2), the covariant derivative $\nabla \tilde{C}$ can be expressed in the following form

$$
\begin{aligned}
\left.\left(\nabla_{W} \tilde{C}\right)(X, Y) Z\right)= & a\left(\nabla_{W} \mathcal{R}\right)(X, Y) Z+b\left[\left(\nabla_{W} S\right)(Y, Z) X\right. \\
& -\left(\nabla_{W} S\right)(X, Z) Y+g(Y, Z)\left(\nabla_{W} Q\right) X \\
& \left.-g(X, Z)\left(\nabla_{W} Q\right) Y\right] .
\end{aligned}
$$

Using (24) in (25) we obtain

$$
\left.\left(\nabla_{W} \tilde{C}\right)(X, Y) Z\right)=a\left(\nabla_{W} \mathcal{R}\right)(X, Y) Z .
$$

Since $a \neq 0$, then in view of the above result we can state the following:

Theorem 4.1. A Kähler-Norden manifold $(M, J, g)$ is quasi-conformally symmetric if and only if it is locally symmetric.

\section{QuASI-CONFORMALlY SEMISYMmetric KÄHLER-NORDEN MANIFOLDS}

In this section we study Quasi-conformally semisymmetric Kähler-Norden manifolds. Assume that R. $\tilde{C}=0$. From (4) we have

$$
\begin{aligned}
\sum_{i} \epsilon_{i} \tilde{C}\left(J e_{i}, J Y\right) e_{i}= & b\left[-2 Q Y-r^{*} J Y\right] \\
& +\frac{r}{n}\left[\frac{a}{n-1}+2 b\right] Y,
\end{aligned}
$$

where $r^{*}$ is the $*$-scalar curvature, which is defined as the trace of $J Q$.

Since $\mathcal{R} . \tilde{C}=0$, then from $(27)$ we have $\mathcal{R} . Q=0$ and hence $\mathcal{R} . S=0$. Again

$$
\begin{aligned}
\tilde{C}(X, Y) Z= & a \mathcal{R}(X, Y) Z+b[S(Y, Z) X-S(X, Z) Y \\
& +g(Y, Z) Q X-g(X, Z) Q Y] \\
& -\frac{r}{n}\left[\frac{a}{n-1}+2 b\right][g(Y, Z) X-g(X, Z) Y],
\end{aligned}
$$

where $a$ and $b$ are constants and $\mathcal{R}, Q$ and $r$ are Riemannain curvature tensor of type $(1,3)$, the Ricci operator defined by $g(Q X, Y)=S(X, Y)$ and the scalar curvature, respectively.

By the $\mathcal{R} . \tilde{C}=0$ and $\mathcal{R} . S=0$ from $(28)$ we have $\mathcal{R} . \mathcal{R}=0$.

Convers by,

$$
\mathcal{R} . \mathcal{R}=0 \Rightarrow \mathcal{R} . S=0 \Rightarrow \mathcal{R} . Q=0 \Rightarrow \mathcal{R} . \tilde{C}=0 .
$$


From the above results we can state the following:

Theorem 5.1. A Kähler-Norden manifold $(M, J, g)$ is quasi-conformally semisymmetric if and only if it is semisymmetric.

In [4], Sluka proved that

Theorem 5.2. [4] A Kähler-Norden manifold $(M, J, g)$ is holomorphically projectively semisymmetric if and only if it is semisymmetric.

In view of Theorems 5.1 and 5.2, we can state the following:

Theorem 5.3. A Kähler-Norden manifold $(M, J, g)$ is quasi-conformally semisymmetric if and only if it is holomorphically projectively semisymmetric.

\section{EXAMPLE}

In [4] Sluka cited an example of a Kähler-Norden manifold which is locally symmetric. This example verifies our Theorem 4.1.

Acknowledgement: The authors are thankful to the referee for his/her comments and valuable suggestions towards the improvement of this paper.

\section{REFERENCES}

[1] A. Borowiec, M. Francaviglia and I. Volovich, Anti-Kählerian manifolds, Diff. Geom. Appl. 12 (2000), 281-289.

[2] G. T. Ganchev and A. V. Borisov, Note on the almost complex manifolds with Norden metric, Compt. Rend. Acad. Bulg. Sci. 39(1986), 31-34.

[3] K. Amur and Y. B. Maralabhavi, On quasi-conformal flat spaces, Tensor, N. S., 31(1977), 194-198.

[4] K. Sluka, On Kähler manifolds with Norden metrics, An. Sÿtiint. Univ. ŤAl.I.CuzaŤ Iasi, Tomul XLVII, S. I. A. Mathematică, 2001, 105-112.

[5] K. Sluka, Properties of the Weyl conformal curvature of Kähler-Norden manifolds, Steps in Diff. Geom., Proc. of the Coll. on Diff. Geom., 2000, Debrecen, Hungary.

[6] K. Yano and S. Sawaki, Riemannian manifolds admitting a conformal transformation group, J. Diff. Geom. 2(1968), 161-184.

[7] K. Yano., Differential geometry of complex and almost complex spaces, Pergamon Press, New York, 1965.

[8] L. P. Eisenhart., Riemannian Geometry, Princeton University Press, 1949.

[9] M. Prvanović., Holomorphically projective mappings onto semisymmetric anti-Kähler manifolds, to appear.

[10] M. Hotloś., On a certain class of Kählerian manifolds, Demonstr. Math. 12(1979), 935-945. 
[11] M. Hotloś., On holomorphically pseudosymmetric Kählerian manifolds, In: Geometry and Topology of Submanifolds, VII, pp. 139-142, World Sci. Publishing, River Edge, N.J., 1995.

[12] Z. Olszak., Bochner flat Kählerian manifolds with a certain condition on the Ricci tensor, Simon Stevin 63(1989), 295-303.

\section{Uday Chand De}

Department of Pure Mathematics

University of Calcutta

35, Ballygaunge Circular Road

Kolkata 700019, West Bengal

INDIA

E-mail address: uc_de@yahoo.com

\section{Pradip Majhi}

Department of Mathematics

University of North Bengal

Raja Rammohunpur, Darjeeling

Pin-734013, West Bengal

INDIA

E-mail address: mpradipmajhi@gmail.com 